\title{
Commentary on: T. Sinnett, N. Karanjia (2009) Decompression of a pneumoperitoneum during a total extraperitoneal (TEP) inguinal hernia repair by incision of the umbilical sac under direct vision
}

\author{
A. Kuthe
}

Received: 4 December 2009/Accepted: 22 December 2009/Published online: 5 February 2010

(C) Springer-Verlag 2010

Since total extraperitoneal (TEP) hernia repair was introduced in the early 1990s, there has been associated discussion regarding pneumoperitoneum. If the latter occurs, and this is not a rare event, the extraperitoneal space can become smaller. This can of course seriously interfere with the TEP procedure and in some cases can necessitate conversion to transabdominal preperitoneal (TAPP) or conventional hernia repair, especially in the early stages of the learning curve.

As a solution to this problem many surgeons use a Verres needle for decompression of the peritoneal space. As Sinnett mentions in his letter to the editor, this method encompasses all the disadvantages and risks of blind peritoneal puncture. In addition, a Verres needle has a maximum flow rate of $21 / \mathrm{min}$. So decompression takes time, or is impossible or unsatisfactory due to the much higher flow rate of any working trocar. Thus, according to in our experience, following a few unsuccessful attempts in 1994, we have never considered a Verres needle as the solution to the problems of pneumoperitoneum.

Sinnett now mentions a new method of decompression by umbilical opening of the peritoneal sac. This of course seems to be safe, and probably gives rise to a better flow. However, due to surface tension, the smaller bubble (extraperitoneal space) will always blow up the bigger one (intraperitoneal space). Thus, in my mind, and in our experience, decompression of the intraperitoneal space does not provide a routine solution to the problem of peritoneal tears in TEP (in our hands close to $50 \%$ of procedures).

We use a different procedure and, since we started with TEP, up to now we have never had a conversion due to pneumoperitoneum in more than 8,000 cases in two different clinics:

1. we work routinely with a pressure of $7 \mathrm{~mm} \mathrm{Hg}$

2. we work in $10-15^{\circ}$ Trendelenburg position

3. we maintain very direct contact with our anaesthesiologists to ensure perfect muscle relaxation until the end of the endoscopic part of the procedure

Adopting just these three simple procedures, in most cases we do not even notice the pneumoperitoneum endoscopically unless pressure is rising due to increasing muscle tension.

The room for TEP is small, and of course easily disturbed by different factors such as pneumoperitoneum or unsatisfactory muscle relaxation. So the way described by Sinnett might be an option to prevent conversion if all other tricks fail.
A. Kuthe $(\square)$

DRK-Krankenhaus Clementinenhaus, Allgemein-, Viszeral- und

Unfallchirurgie, Lützerodestr. 1, 30161 Hannover, Germany

e-mail: akuthe@clementinenhaus.de;

chirurgie@clementinenhaus.de 